\title{
Komplikationen von Magenband versus Magenbypass
}

\author{
Christine Stroh Thomas Manger \\ Klinik für Allgemein-, Viszeral- und Kinderchirurgie, SRH Wald-Klinikum Gera GmbH, Deutschland
}

\section{Schlüsselwörter \\ Bariatrische Chirurgie · Magenband · Magenbypass . \\ Komplikationen · Qualitätssicherung}

\section{Zusammenfassung}

Das Magenband (Gastric Banding, GB) und der Magenbypass (Roux-en-Y-Gastric Bypass, RYGBP) sind die derzeit am häufigsten durchgeführten bariatrischen Operationsverfahren weltweit. Während in den USA das GB die Häufigkeit an RYGBP-Operationen überstiegen hat, zeigt sich in Europa seit mehreren Jahren eine gegenläufige Tendenz. Dies ergibt sich insbesondere aus der Rate an Langzeitkomplikationen nach GB. Früh- und Spätkomplikationen beeinflussen das Outcome der Patienten wesentlich. Daher werden zur Analyse der Komplikationsrate von GB und RYGBP die Daten der Qualitätssicherungsstudie für die operative Therapie der Adipositas ausgewertet und mit Daten der Literatur verglichen.

\section{Einleitung}

Die Adipositas hat sich zu einer weltweiten Epidemie entwickelt. Nach Schätzungen beträgt die Zahl übergewichtiger Erwachsener weltweit 1,7 Milliarden Menschen und die adipöser Erwachsener 312 Millionen [1]. Die Bundesrepublik Deutschland gehört im internationalen und insbesondere im europäischen Vergleich zu den Ländern mit einer sehr hohen Prävalenz der Adipositas. Daten der Mikrozensus-Befragungen des Statistischen Bundesamtes von 1999 bis 2009 haben ergeben, dass die Anzahl der vom Statistischen Bundesamt ausgewiesenen Übergewichtigen (Body Mass Index $(\mathrm{BMI}) \geq 25 \mathrm{~kg} / \mathrm{m}^{2}$ ) in den letzten 10 Jahren um knapp 8\%, die der krankhaft adipösen Deutschen (BMI $\geq 40 \mathrm{~kg} / \mathrm{m}^{2}$ ) aber um $74 \%$ angestiegen ist. Nach den Ergebnissen der Verzehrstudie II des Bundesministeriums für Ernährung und Verbraucherschutz aus

\section{Keywords}

Bariatric surgery - Gastric banding - Gastric bypass . Complications · Quality assurance

\section{Summary}

Complications of Gastric Banding versus Gastric Bypass Gastric banding (GB) and Roux-en-Y gastric bypass (RYGBP) are currently the most-performed bariatric procedures worldwide. GB has become more and more popular in the USA since 2000. In Europe, RYGBP is the procedure of first choice, due to the rate of long-term complications after GB. Early and late postoperative complication rates have a major influence on the patient outcome. Therefore, data of the Nationwide Survey on Bariatric Surgery in Germany were evaluated for complication rate after GB and RYGBP, in comparison with the literature. dem Jahr 2008 liegt der Anteil der krankhaft adipösen Erwachsenen bereits bei 1,5\% der Bevölkerung. Dies entspricht einer Gesamtzahl Betroffener vom rund 1 Million, die einen BMI höher als 40 haben. Die dramatische Gewichtszunahme in der deutschen Bevölkerung geht mit erhöhter Sterblichkeit einher [2].

Mit den seit mehr als 20 Jahren geübten Operationsmethoden des Magenbandes (Gastric Banding, GB) und des Magenbypass (Roux-en-Y-Gastric Bypass, RYGBP) wird das metabolische Syndrom in bis zu $85 \%$ der Fälle effektiv behandelt. Die Auswahl des operativen Verfahrens wird abhängig von der Erfahrung des Chirurgen und den individuellen Patientenkriterien getroffen. In der vorliegenden Arbeit werden die Daten von GB und RYGBP in Bezug auf ihre Komplikationsraten verglichen. Grundlage der Auswertung sind die Daten der Qualitätssicherungsstudie für die operative Therapie der Adipositas.

\section{KARGER}

Fax +497614520714

Information@Karger.de

www.karger.com (c) 2011 S. Karger GmbH, Freiburg

$1662-4025 / 11 / 0047-0024 \$ 38.00 / 0$

Accessible online at:

www.karger.com/ofa
Dr. med. Christine Stroh

Klinik für Allgemein-, Viszeral- und Kinderchirurgie

SRH Wald-Klinikum Gera GmbH

Straße des Friedens 122, 07548 Gera, Deutschland

Tel. +49 365 828-3101, Fax -3102

christine.stroh@wkg.srh.de 


\section{Methoden}

Seit 2005 werden alle bariatrischen Eingriffe wie auch perioperative und späte Ergebnisse der in Deutschland durchgeführten bariatrischen Operationen auf freiwilliger Basis mithilfe der Qualitätssicherungsstudie für die operative Therapie der Adipositas am Institut für Qualitätssicherung in der operativen Medizin gGmbH der Otto-von-Guericke Universität Magdeburg risikoadjustiert erfasst [3, 4]. Die Studie gibt Auskunft zur aktuellen Situation der metabolischen Chirurgie in Deutschland. Sie ermöglicht neben Aussagen zu den Ersteingriffen ebenso Angaben zu Revisionen und Redo-Eingriffen. Frühe und späte Komplikationen sowie deren Häufigkeiten werden in Abhängigkeit vom Operationsverfahren, der Operationsmethode und zahlreicher Begleitvariablen diskutiert. Die Daten der Qualitätssicherungsstudie dienen im Langzeitverlauf dazu, Risikoparameter verschiedener Operationsmethoden zu evaluieren und die Inzidenz von Revisions- und Redo-Eingriffen zu erfassen. Auf Grundlage dieser Daten und der Datenlage der Literatur können patientenspezifische Therapiestrategien festgelegt werden [3].

\section{Ergebnisse}

Derzeit beteiligen sich 54 Kliniken aktiv und freiwillig an der Qualitätssicherungsstudie für die operative Therapie der Adipositas. Im Zeitraum von 2005 bis 2009 wurden 7875 Primäreingriffe erfasst. Das GB $(\mathrm{n}=2437)$ und der RYGBP $(\mathrm{n}=3252)$ sind die im Untersuchungszeitraum am häufigsten durchgeführten bariatrischen Eingriffe.

\section{Demographie}

Bezüglich der Geschlechtsverteilung sind Patienten, die einem GB oder einem RYGBP unterzogen werden, häufiger weiblich. Für das Alter ergeben sich im Vergleich beider Operationsverfahren keine Unterschiede. Patienten mit GB weisen jedoch einen signifikant geringeren BMI auf als Patienten mit RYGBP. Die demographischen Daten sind in Tabelle 1 gezeigt.

\section{Komorbiditäten}

Im Studienzeitraum von 2005 bis 2009 hatten weniger als 17,8\% der Patienten mit GB und 16,8\% der Patienten mit RYGBP keine adipositasassoziierten Begleiterkrankungen. Patienten mit RYGBP (20,4\%) leiden signifikant häufiger als Patienten mit GB (13,0\%) an einem Diabetes mellitus Typ 2. 20,4\% der Patienten mit RYGBP hatten einen nichtinsulinpflichtigen Diabetes mellitus, wohingegen nur $13,0 \%$ der Patienten mit einem GB diesen aufwiesen. Für den insulinpflichtigen Diabetes lag die Relation bei 6,7\% (GB) zu 9,4\% (RYGBP).

Auch für die Hypertonie ergibt sich ein signifikanter Unterschied, mit einer Häufigkeit von 50,6\% für Patienten mit GB und 56,5\% mit RYGBP. Die Inzidenz der Komorbiditäten im Vergleich von GB und RYGBP zeigt Tabelle 2.

Tab. 1. Demographische Parameter 2005-2009

\begin{tabular}{llll}
\hline & \multicolumn{3}{l}{ Geschlechtsverteilung, \% } \\
\cline { 2 - 4 } & Gesamtstudie & GB & RYGBP \\
\hline Männlich & 27,9 & 24,8 & 22,0 \\
Weiblich & 72,1 & 75,2 & 78,0 \\
\hline \multirow{4}{l}{ Mittleres Alter, Jahre } & \\
\cline { 2 - 4 } & Gesamtstudie & GB & RYGBP \\
\hline Männer & 43,3 & 42,5 & 43,3 \\
Frauen & 40,7 & 40,1 & 40,1 \\
Gesamt & 41,4 & 40,7 & 40,8 \\
\hline & Mittlerer BMI, $\mathrm{kg} / \mathrm{m}^{2}$ & \\
\cline { 2 - 4 } & Gesamtstudie & GB & RYGBP \\
\hline Männer & 50,6 & 47,0 & 48,9 \\
Frauen & 48,1 & 45,4 & 48,3 \\
Gesamt & 48,8 & 45,8 & 48,6 \\
\hline
\end{tabular}

Tab. 2. Inzidenz von Komorbiditäten bei GB und RYGBP 2005-2009

\begin{tabular}{lccc}
\hline & Gesamtstudie, \% & GB, \% & RYGBP, \% \\
\hline Keine & 14,0 & 17,8 & 16,8 \\
Hypertonie & 61,3 & 50,6 & 56,5 \\
Kardiovaskuläre Erkrankungen & 10,2 & 8,7 & 7,4 \\
IDDM & 11,1 & 6,7 & 9,4 \\
NIDDM & 20,2 & 13,0 & 20,4 \\
Pulmonal & 16,7 & 14,1 & 17,8 \\
Embolie & 0,6 & 1,7 & 0,5 \\
Schlafapnoe & 20,4 & 13,5 & 19,5 \\
Cholezystolithiasis & 4,4 & 5,2 & 5,4 \\
GERD & 15,4 & 14,5 & 17,8 \\
Varikosis & 5,9 & 7,8 & 4,0 \\
Skeletterkrankungen & 51,5 & 47,0 & 41,4 \\
\hline
\end{tabular}

IDDM = Insulinpflichtiger Diabetes mellitus, NIDDM = nichtinsulinpflichtiger Diabetes mellitus, GERD = gastroesophageal reflux disease. 


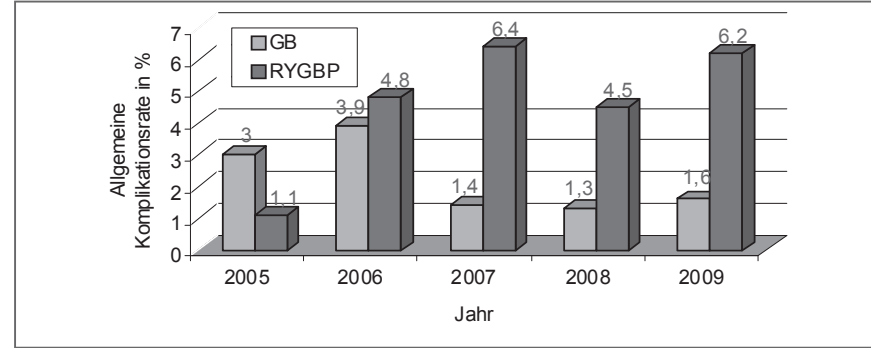

Abb. 1. Vergleich der allgemeinen Komplikationsrate von GB und RYGBP.

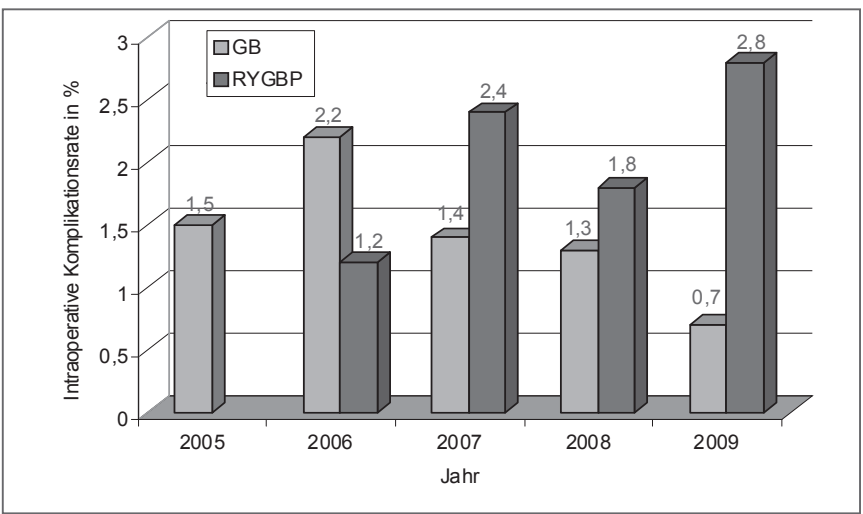

Abb. 2. Vergleich der intraoperativen Komplikationsrate von GB und RYGBP.

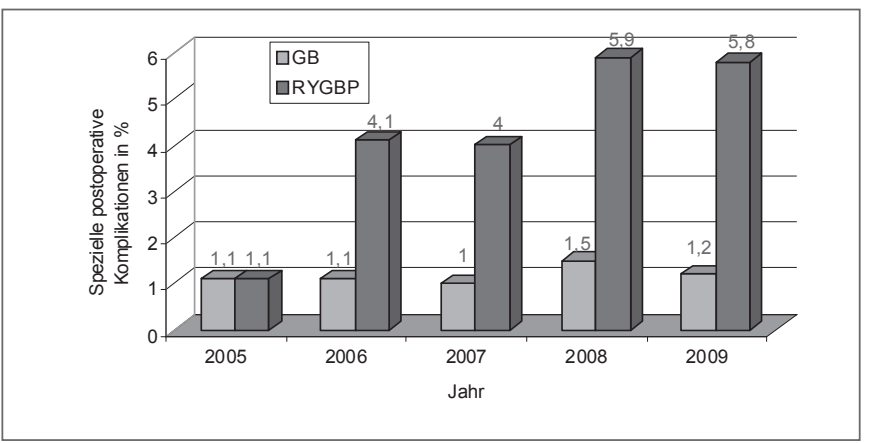

Abb. 3. Vergleich der speziellen Komplikationsrate von GB und RYGBP.

\section{Operationstechnische Daten}

Insbesondere für das GB zeigt sich im Studienverlauf eine deutliche Veränderung der operationstechnischen Details. Während sich die Platzierung des GB in Pars flaccida-Technik ebenso wie die laparoskopische Implantation durchgesetzt hat, werden bei zunehmend mehr Bandplatzierungen keine Serosierungsnähte mehr durchgeführt. In den Jahren 20052007 wurden 9,4\% der Bänder nicht serosiert, in 2008 6,9\% und in $200920,3 \%$.
Tab. 3. Spezielle Komplikationen im Vergleich von GB und RYGBP

\begin{tabular}{llll}
\hline Spezielle Komplikationen & \multicolumn{2}{l}{ Inzidenz, \% } \\
\cline { 2 - 4 } & $2005-2007$ & 2008 & 2009 \\
\hline Blutung & 0,4 & 0,5 & 0 \\
$\quad$ GB & 0,9 & 0,5 & 2,3 \\
$\quad$ RYGBP & & \\
Anastomoseninsuffizienz & & 0,2 & 0 \\
$\quad$ GB & 0,8 & 3,3 & 2,2 \\
$\quad$ RYGBP & & & \\
Anastomosenstenose & & & 0,7 \\
$\quad$ GB & 0,6 & 0,3 & 1,1 \\
$\quad$ RYGBP & & & \\
Ileus & 0,2 & 0,2 & 0,2 \\
$\quad$ GB & 0,5 & 0,4 & 0,5 \\
$\quad$ RYGBP & & & \\
Sepsis & 0,09 & 0 & 0,2 \\
$\quad$ GB & 0,09 & 0,6 & 0,4 \\
$\quad$ RYGBP & & & \\
Peritonitis & 0,09 & 0,2 & 0 \\
$\quad$ GB & 0,09 & 1,1 & 0,9 \\
$\quad$ RYGBP & & & \\
\hline
\end{tabular}

Für den RYGBP zeigt sich die deutliche Zunahme an Kliniken, die den Eingriff anbieten. Die Anzahl der Kliniken stieg von 14 Einrichtungen in 2007 auf 40 im Jahr 2009 an. In dieser Zeit veränderte sich insbesondere die Technik der Durchführung der Gastroenterostomie. Während in den Jahren 2005-2007 56,7\% der Anastomosen in Handnahttechnik erfolgten, wurden 2008 4,9\% und 2009 2,3\% der Anastomosen an der Gastroenterostomie mittels Handnaht durchgeführt. Somit hat sich die Staplertechnik an der Gastroenterostomie wie auch an der Entero-Enterostomie durchgesetzt. Dabei wird die lineare Staplertechnik mit $66,3 \%$ signifikant häufiger eingesetzt als die zirkuläre Technik. Auffallend ist die Abnahme der Häufigkeit des Verschlusses der mesenterialen Bruchpforte. Hier wurde im Zeitraum 2005-2007 noch bei 95,3\% der Eingriffe ein Verschluss durchgeführt. Im Jahr 2009 lag die Rate lediglich bei $62,3 \%$.

\section{Komplikationen}

\section{Allgemeine Komplikationen}

Im Gesamtzeitraum der Studie ist die allgemeine Komplikationsrate nach GB geringer als nach RYGBP. Im Jahr 2005 fand sich eine höhere allgemeine Komplikationsrate nach GB als nach RYGBP, was jedoch darauf zurückzuführen ist, dass der RYGBP zu diesem Zeitpunkt nur in wenigen Kliniken mit hoher Expertise erfolgte. Seither liegt die allgemeine Komplikationsrate für den RYGBP zwischen 4,5\% (2008) und 6,4\% (2008) (Abb. 1). Häufige allgemeine Komplikationen sind Fieber $(1,9 \%)$, pulmonale $(1,3 \%)$ und renale Komplikationen $(0,5 \%)$ sowie Harnwegsinfekte $(0,7 \%)$. 
Tab. 4. Mortalität im Vergleich mit der Metaanalyse

\begin{tabular}{lllllll}
\hline & \multicolumn{2}{l}{ Qualitätssicherungsstudie } & $2005-2009$ & \multicolumn{3}{c}{ Metaanalyse Buchwald et al. [9] } \\
\cline { 2 - 7 } & Gesamtstudie & GB & RYGBP & & GB & RYGBP \\
\hline Todesfälle, \% & 0,33 & 0 & 0,3 & 0,4 & 0,1 & 0,3 \\
\hline
\end{tabular}

Tab. 5. Demographie im Vergleich mit randomisierten Studien

\begin{tabular}{llllc}
\hline & \multicolumn{2}{l}{$\begin{array}{l}\text { Boza et al. [8], } \\
\text { 2001-2003 }\end{array}$} & \multicolumn{2}{l}{$\begin{array}{l}\text { Nguyen et al. [10], } \\
\text { 2002-2007 }\end{array}$} \\
\cline { 2 - 5 } & GB & RYGBP & GB & RYGBP \\
\hline Anzahl der Patienten & 62 & 91 & 86 & 111 \\
Alter, Jahre & 38,4 & 34,5 & 45,8 & 41,4 \\
BMI, kg/m & 35,8 & 39,6 & 45,5 & 47,5 \\
\hline
\end{tabular}

\section{Intraoperative Komplikationen}

Die intraoperative Komplikationsrate für den RYGBP ist in den letzten Jahren angestiegen. Sie lag im Jahr 2009 bei 2,8\% und ist damit signifikant höher als die Komplikationsrate für das GB (0,7\%) (Abb. 2). Ursachen intraoperativer Komplikationen beim GB waren Blutungskomplikationen infolge von Gefäßverletzungen und Milzläsionen. Für den RYGBP liegt die Rate an Milzläsionen im Gesamtzeitraum bei 0,25\%. Die hohe Rate an intraoperativen Komplikationen beim RYGBP resultiert auch aus 1,5\% technischen Problemen mit Staplern und Klammernahtinstrumenten.

\section{Spezielle Komplikationen}

Die spezielle Komplikationsrate nach RYGBP ist im Gesamtzeitraum signifikant höher als die nach GB und ist in Abbildung 3 gezeigt. Die Unterschiede in der Inzidenz spezieller Komplikationen ergeben sich aus dem Risiko von Anastomosenkomplikationen nach RYGBP mit nachfolgenden septischen Komplikationen (Tab. 3).

\section{Mortalität}

Die Mortalität für das GB liegt in der Qualitätssicherungsstudie bei $0 \%$ und für den RYGBP bei $0,3 \%$ (Tab. 4 ).

\section{Diskussion}

Die vorliegenden prospektiven Studien zum GB und RYGBP untersuchen weder den Einfluss des Geschlechts, des BMI, des Alter und der Komorbiditäten auf die perioperative Komplikationsrate noch auf das Outcome. Die demographischen Daten unterscheiden sich signifikant von den Daten der Qualitätssicherungsstudie (Tab. 5).

Das GB ist ein effektives und wenig invasives Verfahren. Für das Outcome der Patienten sind nach GB insbesondere die Langzeitkomplikationen von Bedeutung. Aufgrund der unterschiedlichen Operationstechniken und Bandtypen existieren in der Literatur hierzu nur sehr heterogene Daten aus SingleCenter-Studien, die vereinzelt Follow-up-Daten mit einer Nachsorgezeit von über 10 Jahren publiziert haben [5]. Fehlende operationstechnische Details und Angaben zur postoperativen Strategie der Bandjustierung sowie der Art und Weise der Patientennachsorge führen dazu, dass diese Studien bezüglich ihrer Angaben zu Komplikationen nicht vergleichbar sind.

Anhand der Daten der Literatur ist jedoch bekannt, dass das GB in exakter Pars-flaccida-Technik durchgeführt werden sollte. Ein Einfluss des Bandtyps auf die Komplikationsrate konnte anhand der vorliegenden Literaturanalyse und in Studien [6] nicht nachgewiesen werden. Ursachen hierfür sind die Heterogenität der Daten und der bestehende Einfluss der Technik der Bandplatzierung auf die Komplikationsrate. Bezüglich der Serosierung des Magenbandes liegen derzeit keine Daten aus prospektiv randomisierten Studien vor. Daher sollte anhand detaillierter Daten der Qualitätssicherungsstudie in den folgenden Jahren der Zusammenhang von Bandtyp und Operationstechnik mit den Komplikationsarten und -raten mithilfe von Propensity Score-Analysen untersucht werden [4].

Der RYGBP ist ein Verfahren, das aufgrund der hohen Rate an Komorbiditäten der Patienten in Deutschland zunehmend Verbreitung findet. Intra- und frühe postoperative Komplikationen wie Anastomoseninsuffizienz, -stenose und Ulzera an der Gastroenterostomie beeinflussen das Outcome wesentlich.

Die aus den Daten der Qualitätssicherungsstudie resultierende Insuffizienzrate an der Gastroenterostomie und die Stenoserate liegen unter den in der Literatur publizierten Daten. Hier werden Insuffizienzraten von bis zu 5,1\% berichtet [7]. Eine Ursache hierfür ist die hohe Fallzahl der RYGBP in insgesamt wenigen Kliniken. Hierfür spricht der Anstieg der intra- und postoperativen Komplikationsrate in 2008 und 2009. Seither wird der RYGBP zunehmend als Standardverfahren eingesetzt. Anhand der Literatur kann keine eindeutige Empfehlung zur Reduktion der Insuffizienzrate durch eine bestimmte Technik der Anastomosierung (Handnaht, Zirkulär- oder Linearstapler) gegeben werden. Dies gilt auch für die Daten der Qualitätssicherungsstudie, die die Handnaht und Staplernaht bei der Anlage der Gastroenterostomie vergleichen. Ein Einfluss von Helicobacter-pylori-Infektionen auf die Inzidenz von Anastomosenkomplikationen ist in der Literatur und anhand der vorliegenden Daten der Qualitätssicherungsstudie nicht ausreichend untersucht. Gemäß unserer Studiendaten erfolgt bei $87,9 \%$ der Patienten präoperativ eine Gastroskopie. 
Die Inzidenz innerer Hernien ist in der vorliegenden Qualitätssicherungsstudie signifikant niedriger als in den anhand der Literatur ermittelten Daten (0,03\% vs. $2,83 \%$; $p<0,001)$. Dieses ist im Zusammenhang mit der antekolisch-antegastrischen Technik sowie mit dem Verschluss der inneren Bruchpforten in 88,5\% der Eingriffe zu sehen. Entsprechend der Literaturangaben geht beides mit einer geringeren Inzidenz von inneren Hernien einher.

Für den RYGBP zeigt sich eine deutliche Veränderung der technischen Durchführung der Gastroenterostomie. Allerdings existieren dazu keine Daten aus prospektiv randomisierten Studien mit hoher Fallzahl. Der fehlende Verschluss der mesenterialen Bruchlücken korreliert mit einer zunehmenden Inzidenz an inneren Hernien unter Berücksichtigung des Zusammenhanges mit der Technik der ante- versus retrokolischen Anastomosierung.

Die Evaluierung patientenspezifischer Parameter und der Einfluss verschiedener operativer Techniken auf das Outcome der Patienten sind im Langzeitverlauf anhand der Daten der Qualitätssicherungsstudie zu analysieren, um die frühen und späten postoperativen Komplikationsraten beeinflussen zu können.

\section{Schlussfolgerungen}

Für die positiven Langzeiteffekte der Gewichtsreduktion mit der effektiven Behandlung des metabolischen Syndroms und der Verbesserung der Lebensqualität liegen evidenzbasierte Daten vor. Dagegen ist nur ein geringer Anteil klinischer und chirurgischer Standards evidenzbasiert und anhand randomisierter Studien abgesichert.
Aufgabe der Qualitätssicherungsstudie ist es daher, den Einfluss der Veränderungen der Operationstechnik sowohl beim GB als auch beim RYGBP auf die Komplikationsrate im weiteren Verlauf zu untersuchen.

Die vorliegenden prospektiven Studien zum GB und RYGBP untersuchen weder den Einfluss des Geschlechts, des BMI, des Alters und der Komorbiditäten auf die perioperative Komplikationsrate noch auf das Outcome. Studien zum GB zeigen eine Reoperationsrate von 3-4\% pro Jahr der Nachsorge, mit bis zu 24\% nach 5 Jahren [5, 8]. Für den RYGBP beträgt die Reoperationsrate nach 5 Jahren bis zu 7,2\% bei insgesamt erhöhter perioperativer Morbidität und Mortalität. Daher ist die Wahl des geeigneten Operationsverfahrens immer eine Einzelfallentscheidung. Sie ist abhängig von patientenspezifischen Parametern und Risiken, dem beruflichen und sozialen Umfeld des Patienten und der Expertise der bariatrischen Einrichtung.

\section{Dank}

Wir danken folgenden Firmen für die finanzielle Unterstützung der Studie: Johnson and Johnson MEDICAL GmbH; Ethicon Endo-Surgery Deutschland, Norderstedt; Covidien Deutschland GmbH, Neustadt/ Donau; Lamed GmbH, München-Oberhaching; Pharm-Allergan GmbH, Ettlingen; Sanofi-Aventis Deutschland GmbH, Berlin; W.L. Gore Associates $\mathrm{GmbH}$, Putzbrunn.

\section{Disclosure Statement}

Es besteht kein Interessenkonflikt. Die korrespondierende Autorin versichert, dass keine Verbindungen mit der Firma, deren Produkt im Artikel genannt ist, oder einer Firma, die ein Konkurrenzprodukt vertreibt, bestehen. Die Präsentation des Themas ist unabhängig und die Darstellung der Inhalte produktneutral.

\section{Literatur}

1 Deitel M: Overweight and obesity worldwide now estimated to involve 1.7 billion people. Obes Surg 2003;13:329-330.

2 Weiner RA, Stroh C: Metabolische Chirurgie-Update 2011. CHAZ 2010;11:587-599.

3 Stroh C, Manger T: Studie zur Qualitätskontrolle der operativen Therapie der Adipositas. Mitt Dtsch Ges Chir 2004;33:389-391.

${ }_{4}$ Stroh C, Birk D, Flade-Kuthe R, Franken M, Herbig B, Höhne S, Köhler H, Lange V, Ludwig K, Matkowitz R, Meyer G, Meyer F, Pick P, Horbach T, Krause S, Schäfer L, Schlensak M, Shang E, Sonnenberg T, Susewind M, Voigt H, Weiner R, Wolff S, Lippert H, Wolf AM, Schmidt U, Manger T; Bariatric Surgery Working Group: A nationwide survey on bariatric surgery in Germany - results 2005-2007. Obes Surg 2009;19:105-112.
5 Suter M, Calmes JM, Paroz A, Giusti V: A 10-year experience with laparoscopic gastric banding for morbid obesity: high long-term complication and failure rates. Obes Surg 2006;16:829-835.

6 Fried M, Miller K, Kormanova K: Literature review of comparative studies of complications with Swedish Band and Lap-Band ${ }^{\circledR}$. Obes Surg 2004;14: 256-260.

7 Sánchez-Santos R, Vilarrasa N, Pujol J, Moreno P, Manuel Francos J, Rafecas A, Masdevall C: Is Roux-en-Y gastric bypass adequate in the superobese? Obes Surg 2006;16:478-483.

8 Boza C, Gamboa C, Awruch D, Perez G, Escalona A, Ibañez L: Laparoscopic Roux-en-Y gastric bypass versus laparoscopic adjustable gastric banding: five years of follow-up. SOARD 2010;6:470-475.
9 Buchwald H, Estok R, Fahrbach K, Banel D, Sledge I: Trends in mortality in bariatric surgery: a systematic review and meta-analysis. Surgery 2007;142:621-632; discussion 632-635.

10 Nguyen NT, Slone JA, Nguyen XM, Hartman JS, Hoyt DB: A prospective randomized trial of laparoscopic gastric bypass versus laparoscopic adjustable gastric banding for the treatment of morbid obesity: Outcomes, quality of life, and costs. Ann Surg. 2009;250:631-641. 